\title{
Swiss Quality Award 2011 - Die Gewinner (Teil 1)
}

Aus über 100 eingereichten Arbeiten wurden anlässlich des Nationalen Symposiums für Qualitätsmanagement im Gesundheitswesen vier Projekte mit dem Swiss Quality Award 2011 ausgezeichnet. Die drei Trägerorganisationen IEFM, SQMH und die FMH freuen sich, die Gewinnerprojekte der Kategorien «Patientensicherheit» und «Information» vorzustellen. Mehr über die Siegerprojekte der Kategorien «Management» und «Technologie» erfahren Sie in der kommenden Ausgabe der Schweizerischen Ärztezeitung.

\section{Gewinner Kategorie Patientensicherheit}

Martin Eggera,

Florian Balmera,

Esther M. Näfa,

Heidi Friedli-Wüthricha,

Kathrin Mühlemann ${ }^{b}$

a Regionalspital Emmental

b Institut für Infektionskrankheiten, Universität Bern
Korrespondenz:

Martin Egger

Regionalspital Emmental

Oberburgstrasse 54

CH-3400 Burgdorf

Tel. 0344212300

martin.egger@rs-e.ch

\section{Reduktion von Urinkathetertagen und Antibiotika-Behandlungstagen für Harn- wegsinfektionen in der stationären Akut- medizin}

Nosokomiale Infektionen gefährden die Patientensicherheit im Spital. Harnwegsinfektionen (HWI) sind die häufigsten und am leichtesten vermeidbaren unter ihnen [1]. Der Blasenkatheter ist der wichtigste Risikofaktor für die Entwicklung von nosokomialen HWI [2]. Zahlreiche Blasenkatheter werden ohne adäquate Indikation eingelegt und viele zu spät entfernt. Das grosse Präventionspotential des Verzichts auf unnötige Blasenkatheter und überflüssige Antibiotikatherapien von HWI liegt in vielen Spitälern brach. Hier kann im Sinne des noch jungen Ansatzes der quartären Prävention, d.h. der Vermeidung von Überbehandlung durch Verzicht, viel geleistet werden für die Patientensicherheit in der stationären Akutmedizin.

Die Autorinnen und Autoren haben sich zum Ziel gesetzt, die Urinkathetertage und die Antibiotika-Behandlungstage für HWI in der Medizinischen Klinik des Regionalspitals zu reduzieren und diese Reduktion solide zu dokumentieren.

Nach der Messung und Dokumentation des IstZustandes wurde für die Zielerreichung eine multimodale Intervention durchgeführt, die folgende Massnahmen umfasste:

- Feedback der Daten der Messperiode vor der Intervention an die involvierten Abteilungen;

- Überarbeitung bestehender Richtlinien zur Therapie von HWI

- Erarbeitung von Richtlinien zur Diagnostik von HWI und zum Management von Blasenkathetern in einer interprofessionellen Kooperation von Pflege und Ärzteschaft;

- Einführung einer Positiv- und einer Negativ-Liste von Katheterindikationen; Implementierung im

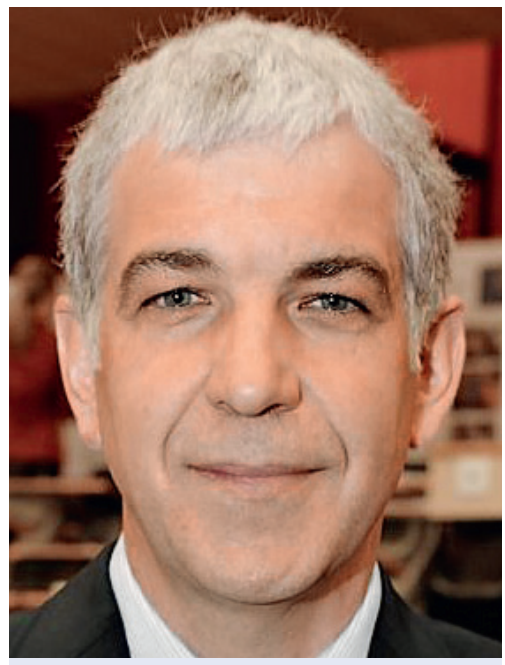

Martin Egger hat als Vertreter seines Teams den Preis in der Kategorie «Patientensicherheit» entgegengenommen.

Rahmen eines Kathetermeldeformulars im Intranet;

- Absolvierung von uricheck (selbstentwickeltes, webbasiertes Lerninstrument für rationale Antibiotikatherapien bei HWI) durch die Ärztinnen und Ärzte;

- Einführung eines Erinnerungsklebers für die Patientendokumentation bei Katheterträgern;

- Weiterbildungen für Pflegefachpersonen und Ärzteschaft.

Eine erneute Erhebung der Messgrössen nach der Intervention zeigte auf, dass diese auf einfachen Massnahmen basierende, aber multimodal und interprofessionell konzipierte Intervention Urinkathetertage und Antibiotika-Behandlungstage für asymptomatische Bakteriurien bei internmedizinischen Patienten 
Abbildung 1

Wichtigste Zielgrössen vor und nach der Intervention.

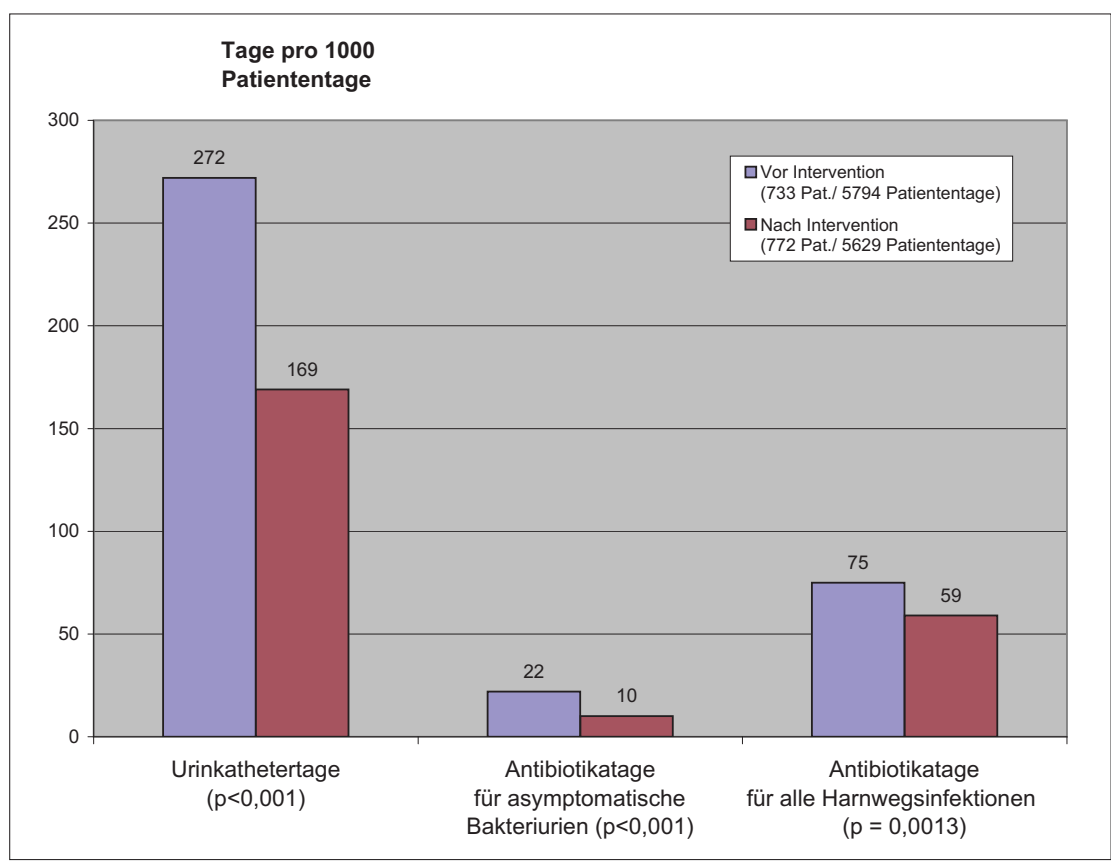

signifikant um 38 bzw. 55 Prozent reduzieren konnte. Der Verzicht auf Überflüssiges leistet in diesem Bereich für die Patientensicherheit mehr als manche teure technische Innovation und ist damit ein gutes Anwendungsbeispiel für den Ansatz der quartären Prävention. Mittels Wiederholung von Elementen der Intervention soll die Nachhaltigkeit der Qualitätsverbesserung gewährleistet werden. Weiter ist im Regionalspital Emmental eine Ausweitung auf die chirurgischen Disziplinen geplant.

\section{Literatur}

1 Umscheid CA et al. Estimating the proportion of healthcare-associated infections that are reasonably preventable and the related mortality and costs. Infect Control Hosp Epidemiol. 2011;32:101-14.

2 Hooton TM et al. Diagnosis, prevention, and treatment of catheter-associated urinary tract infection in adults: 2009 international clinical practice guidelines from the Infectious Diseases Society of America. Clin Infect Dis. 2010;50:625-63.

\section{Gewinner Kategorie Information}

\begin{abstract}
Margareta Schmid, Ruedi Hösli, Stefan Neuner, Ueli Grüninger
\end{abstract}

Kollegium für Hausarztmedizin KHM, Bern

Korrespondenz:

Ueli Grüninge

Kollegium für Hausarztmedizin

Landhausweg 26

CH-3007 Bern

Tel. 0313700671

ueli.grueninger@hin.ch

www.

gesundheitscoaching-khm.ch

\section{Gesundheitscoaching - Patient und Arzt als Partner: ein Programm zur Erhöhung der Prozess- und Resultatqualität bei Gesund- heitsförderung und Prävention in der Haus- arztpraxis}

Prävention und Gesundheitsförderung in der Sprechstunde sind wichtige Aufgaben für den Hausarzt. Das Projekt «Gesundheitscoaching» (www.gesundheitscoaching-khm.ch) hat zum Ziel, Gesundheitsförderung und Prävention in Arztpraxis und Gesundheitswesen nachhaltig zu etablieren, eine patientenzentrierte Beratungskultur zu fördern und dafür günstige methodische, strukturelle und finanzielle Kontextbedingungen zu schaffen.

Das Projekt des Kollegiums für Hausarztmedizin KHM [1] beruht auf einem partnerschaftlichen Verhältnis zwischen Patient und Arzt und unterstützt beide Seiten dabei, gemeinsam und effizient die Gesundheit des Patienten zu stärken und Krankheiten vorzubeugen. Im Zentrum stehen Verhaltensweisen wie Rauchen, Alkoholkonsum, Bewegungsmangel, Übergewicht, Ernährung und Stress. Das Gesundheitscoaching unterstützt den Patienten dabei, seine Erfahrungen mit dem Wissen des Arztes zu kombinieren, um erfolgreich und nachhaltig sein Gesundheitsverhalten positiv zu beeinflussen [2].

Bei Projekten im Bereich sprechstundenbasierte Gesundheitsförderung und Prävention wird aus inhaltlicher Sicht oft bemängelt, dass

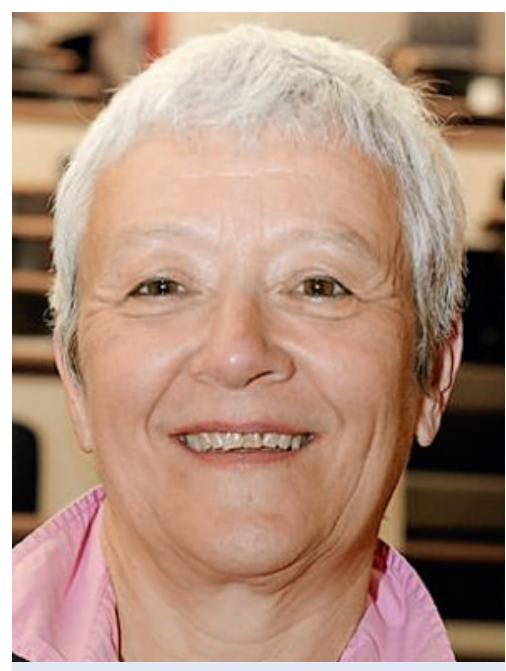

Margareta Schmid präsentierte im Namen des Projektteams das Siegerpojekt in der Kategorie «Information».

- die existierenden Interventionsangebote fast durchwegs nur auf einzelne Gesundheitsverhaltensweisen bzw. Risikofaktoren fokussieren,

- diese weder methodisch noch strategisch untereinander koordiniert sind und deshalb weder auf die Bedürfnisse des Patienten noch auf die Praxisrealität des Arztes eingehen, 
- das traditionelle ärztliche Beratungsvorgehen vorwiegend direktiv und präskriptiv-verordnend ist und deshalb die zentrale Rolle des Patienten vernachlässigt.

Das Projekt Gesundheitscoaching behebt diese Schwächen durch innovative Kombination der folgenden drei Dimensionen:

\section{Patientenorientierung}

Das Gesundheitscoaching holt den einzelnen Patienten bei dessen individueller Verhaltenskonstellation ab. Es erarbeitet mit ihm zuerst seine Prioritäten und Motivationen und ermöglicht so eine nachhaltige Mobilisierung der individuellen Gesundheitskompetenz und Ressourcen.

\section{Strategisch-inhaltliche Koordination}

Das Gesundheitscoaching integriert die bisher existierenden zahlreichen, auf ein bis maximal zwei Gesundheitsverhaltensweisen fokussierten Interventionsangebote in einen einheitlichen, faktorenübergreifenden, d.h. polyvalent anwendbaren Approach.

\section{Methoden-Integration}

Das Gesundheitscoaching bietet zu diesem Zweck dem Arzt einfach anwendbare Algorithmen für die Sprechstunde an, mit deren Hilfe er die Patienten auf ihr Gesundheitsverhalten anspricht, sensibilisiert und motiviert, damit diese ihre eigenen Ziele und Massnahmen entwickeln und umsetzen können, begleitet von ihrem Arzt. Das Gesundheitscoaching operationalisiert in dieser Form erstmals moderne Gesundheitsförderungs- und Beratungskonzepte (u.a. Gesundheitskompetenz, Empowerment, Shared Decision Making, Motivational Interviewing) und nutzt so das Potential dieser Konzepte.

Im Projekt Gesundheitscoaching bekommt der Patient die Hauptrolle. Er entwickelt sein eigenes Gesundheitsprojekt und setzt es eigenständig um, wobei er sich die individuell nötige Unterstützung in $\mathrm{Zu}$ sammenarbeit mit dem Arzt holt, statt in der passiven Rolle des zu Behandelnden zu verbleiben. Das Gesundheitscoaching macht gleichzeitig den Arzt zum Coach des Patienten. Der Arzt fördert und mobilisiert beim Patienten dessen Gesundheitskompetenzen, Ressourcen und Eigenverantwortung, statt direktivpaternalistisch Ratschläge und Rezepte zu geben.

Das Gesundheitscoaching spielt sich sowohl in der Sprechstunde als auch im Alltag des Patienten ab. So bearbeiten Arzt und Patient in der Sprechstunde jeweils einen Teilschritt Richtung Gesundheitsverhaltens-Änderung. Im Alltag arbeitet der Patient am Besprochenen und setzt es Schritt für Schritt um. Dieser Zwei-Takt führt das Team Patient/Arzt Schritt für Schritt, in simplen Algorithmen vom Allgemeinen

\section{Swiss Quality Award - Innovations in Healthcare}

Der Swiss Quality Award steht für Engagement im Gesundheitswesen. Er zeichnet Innovationen aus, die neue Akzente im Qualitätsmanagement setzen. Im Zentrum stehen Pionierinnen und Pioniere, die mit viel Kreativität und noch mehr Arbeit neue Ideen entwickeln und umsetzen, um die Qualität im Gesundheitswesen nachhaltig positiv zu beeinflussen. Der Preis wird in den vier Kategorien Management, Patientensicherheit, Technologie und Information verliehen und von der Schweizerischen Gesellschaft für Qualitätsmanagement im Gesundheitswesen (SQMH), dem Institut für Evaluative Forschung in der Medizin (IEFM) der Universität Bern sowie der Verbindung der Schweizer Ärztinnen und Ärzte (FMH) getragen. Alle Informationen zum Swiss Quality Award sowie eine Onlineplattform mit den Projektpostern der Gewinner und weiteren Projekten befinden sich unter www.swiss qualityaward.ch

zum Speziellen: zuerst Sensibilisierung/Motivation, dann Auswahl von und Einigung auf ein neues Zielverhalten, und schliesslich Planung und Umsetzung eines persönlichen Gesundheitsprojekts.

Das Beratungsprogramm Gesundheitscoaching (Abläufe, Instrumente sowie Schulung und Praxissupport) wurde im Jahr 2010 während 12 Monaten im Massstab 1:1 in 20 Arztpraxen des Kantons St. Gallen erprobt. In dieser Zeit sprachen die Ärzte insgesamt über 1000 Patientinnen und Patienten an.

Die vorläufigen Resultate der noch laufenden Prozess- und Resultat-Evaluation zeigen, dass die Beteiligung grösser als erwartet ist und die Patienten die Gelegenheit sowie das Angebot begrüssen. Aufgrund der Ergebnisse und Erfahrungen aus dem Pilotprojekt soll das Programm Gesundheitscoaching auf sprachregionaler und nationaler Ebene verbreitet werden.

\section{Referenzen}

1 Das KHM ist die Schweizerische Stiftung zur Förderung der Medizinischen Grundversorgung (Träger: Schweizerische Gesellschaft für Allgemeinmedizin SGAM, Schweizerische Gesellschaft für Allgemeine Innere Medizin SGIM, Schweizerische Gesellschaft für Pädiatrie SGP, Schweizerische Akademie der Medizinischen Wissenschaften SAMW und Medizinische Fakultäten der Universitäten Basel, Bern, Genf, Lausanne, Zürich).

2 vgl. Projektbeschreibung: Grüninger U. et al. Gesundheitscoaching: ein Programm für Gesundheitsförderung und Prävention in der Hausarztpraxis vom Kollegium für Hausarztmedizin (KHM). Schweiz Ärztezeitung. 2009;90(45):1729-32. 